\title{
ON SEMIBOUNDED DIFFERENTIAL OPERATORS
}

\author{
HARRY HOCHSTADT ${ }^{1}$
}

\begin{abstract}
ABSTRAC. 1 . It is shown that regular ordinary differential operators have a semibounded spectrum. The proof requires fewer prerequisites than other proofs found in the literature and also yields estimates on the lower bound of the spectrum.
\end{abstract}

The purpose of this note is to provide a short and relatively elementary proof of the following theorem.

THEOREM. Let $p_{0}(x), p_{1}(x), \cdots, p_{n}(x)$ be a set of real functions on $[0,1]$, such that $p_{k}(x) \in C^{k}[0,1]$ for $k=0,1, \cdots, n$. Let $\left\{a_{k}\right\}$ denote their lower bounds such that $p_{k}(x) \geqq a_{k}$. In particular $p_{n}(x)$ is to be positive so that $p_{n}(x) \geqq a_{n}>0$. Define the formally selfadjoint differential operator $l$ by

$$
l(u)=\sum_{k=0}^{n}(-1)^{k}\left(p_{k}(x) u^{(k)}\right)^{(k)}
$$

$l$ is defined on a suitable domain in $L_{2}[0,1]$.

We now consider the boundary conditions

$$
\sum_{j=0}^{2 n \cdots 1} \alpha_{i j} u^{(j)}(0)+\sum_{j=0}^{2 n-1} \beta_{i j} u^{(j)}(1)=0, \quad i=1,2, \cdots, 2 n .
$$
have

We assume that for all functions $u, v \in C^{2 n}[0,1]$ that also satisfy (2) we

$$
(l u, v)-(u, l v)=\int_{0}^{1}[(l u) \bar{v}-u(\overline{l v})] d x=0 .
$$

Let $L_{1}$ denote the regular selfadjoint operator generated by land the boundary conditions (2) on the Hilbert space $L_{2}[0,1]$. The spectrum of $L_{1}$ is bounded below.

For other proofs of this theorem see [1], [2], [3]. The proof of the theorem will be based on the following lemma.

Received by the editors January 29, 1972.

AMS 1970 subje't classifications. Primary 34B25, 47B25; Secondary 47E05.

1 The author gratefully acknowledges the support of the National Science Foundation under grant GP-27960.

c) American Mathematical Society 1972 
Lemma. Let $l$ be as in the above theorem. Consider the boundary conditions

$$
u^{(j)}(0)=0, \quad u^{(j)}(1)=0, \quad j=0,1, \cdots, n-1 .
$$

Denote by $L_{0}$ the regular selfadjoint operator generated $b y l$ and the boundary conditions (4). The spectrum of $L_{0}$ is bounded below.

Proof of The Lemma. By integration by parts, use of the boundary conditions (4) and the fact that all $p_{k}(x) \geqq a_{k}$ we have

$$
\left(L_{0} u, u\right)=\int_{0}^{1} \sum_{k=0}^{n} p_{k}\left|u^{(k)}\right|^{2} d x \geqq \int_{0}^{1} \sum_{k=0}^{n} a_{k}\left|u^{(k)}\right|^{2} d x .
$$

In order for $u$ to be in the domain of $L_{0}$ it must have at least $n$ continuous derivatives. We can therefore expand $u$ in a Fourier series that can be differentiated term by term $n$ times. Then

$$
u=\sum_{m=1}^{\infty} b_{m} \sin m \pi x
$$

and inserting the above in (5) we have

$$
\left(L_{0} u, u\right) \geqq \frac{1}{2} \sum_{m=1}^{\infty}\left|b_{m}\right|^{2} \sum_{k=0}^{n} a_{k}(m \pi)^{2 \mu} .
$$

We now define the polynomial $q(x)=\sum_{k=0}^{n} a_{k} x^{2 k}$ and find, using (7), that

$$
\left(L_{0} u, u\right) \geqq \frac{1}{2} \min _{m} q(m \pi) \sum_{m=1}^{\infty}\left|b_{m}\right|^{2}=\min _{m} q(m \pi)(u, u) .
$$

Since $a_{n}>0, q(x)$ must have a greatest lower bound so that the conclusion of the lemma follows from (8).

N.B. (8) yields a lower bound for the smallest eigenvalue of $L_{0}$.

Proof of THE THEOREM. We assume that 0 is not an eigenvalue for $L_{1}$ or $L_{0}$ and that in fact $L_{0}$ is a positive definite operator. Otherwise we can shift $L_{1}$ and $L_{0}$ by a constant to accomplish this. Now we can associate a Green's function with both operators. Denote these by $K_{1}(x, y)$ and $K_{0}(x, y)$ respectively. These satisfy the equations

$$
\begin{aligned}
& L_{0} K_{0}(x, y)=\delta(x-y), \\
& L_{1} K_{1}(x, y)=\delta(x-y) .
\end{aligned}
$$

But it is evident since both have the same singular behavior at $x=y$, that

$$
l\left(K_{\mathrm{i}}(x, y)-K_{0}(x, y)\right)=0 .
$$


(11) is in fact an ordinary differential equation so that

$$
K_{1}(x, y)-K_{0}(x, y)=\sum_{j=1}^{2 n} c_{j}(y) u_{j}(x)
$$

where the $u_{j}(x)$ are $2 n$ linearly independent solutions of $l u=0$, and the $c_{j}(y)$ suitable functions of $y$. Since $K_{0}(x, y)$ and $K_{1}(x, y)$ are symmetric in $x$ and $y$ each $c_{j}(y)$ is expressible in terms of the $u_{j}(y)$ so that (12) can be rewritten as

(13) $K_{1}(x, y)-K_{0}(x, y)=\sum_{i, j=1}^{2 n} \gamma_{i j} u_{i}(y) u_{j}(x)=D(x, y), \quad \gamma_{i j}=\gamma_{j i}$.

Let $K_{1}, K_{0}, D$ denote integral operators associated with the above kernels. Then

$$
\left(K_{1} u, u\right)=\left(K_{0} u, u\right)+(D u, u) \geqq(D u, u)
$$

since $K_{0}$ is positive definite. $D$ is a degenerate operator and therefore semibounded so that (14) proves the theorem.

N.B. (14) shows that $K_{1}$ has at most $2 n$ negative eigenvalues.

\section{REFERENCES}

1. N. Dunford and J. T. Schwartz, Linear operators. II: Spectral theory, selfadjoint operators in Hilbert space, Interscience, New York, 1963. MR 32 \#6181.

2. M. A. Naǐmark, Linear differential operators. Vol. II, GITTL, Moscow, 1954; English transl., Ungar, New York, 1967, pp. 93 ff. MR 16, 702; MR 41 \#7485.

3. H. Widom, Lectures on integral equations, Van Nostrand Math. Studies, no. 17, Van Nostrand Reinhold, New York, 1969, pp. 73 ff. MR 39 \#4621.

Departmfnt of Mathematics, Polytechnic Institute of Brooki.yn, Brooklyn, NEW YORK 11201 